\title{
Nursing Homes
}

\section{OP 039 EVALUATING A SPECIALIST PALLIATIVE CARE} INITIATIVE FOR NURSING AND RESIDENTIAL HOMES

Sarah Locker, Sarah Callin. Saint Catherine's Hospice, Scarborough, UK

10.1136/bmjspcare-2014-000654.39

Background The End of Life Care report from the National Audit Office identified that $50 \%$ of care home residents who died in hospital, could have died in their care home. The support needed by care homes to enable residents to remain in their preferred place for death is complex; education alone does not change practice. We report interim data from a two year pilot of a hospice care home support team. 
Aims To evaluate the effect of the care home support team on palliative care outcomes.

Method The intervention. A team of three (two whole time equivalent) palliative care nurse specialists work with six nursing and four residential homes. They review systems of care, implement good-practice guidance and deliver an education programme. Ad hoc clinical access and a regular clinical forum provide on-going support. Data collection (September 2012-end August 2013). Baseline retrospective data for the year prior to service introduction has been collected from the pilot care homes for the following outcomes: hospital admission, death in hospital, preferred place documentation, actual and preferred place of death. Measures repeated at one year.

Results Data from 10 care homes has been collected. Compared with baseline data, the following outcomes have improved for residents in pilot care homes: number of hospital admissions per annum (baseline; 146, review 70); deaths in hospital/all deaths per annum (baseline 15/81, 19\%; review 18/134, $13 \%$ ); preferred place of death documented (baseline 45/81, 56\%; review 100/134, 75\%); concordance with preference (baseline 89\%; review 97\%). Questionnaire feedback from residents, families and professionals involved with the service has also been positive.

Conclusions This pilot data indicates that focused education and clinical support by a hospice care home support team is an effective way of improving quality of palliative care provision in nursing and residential homes. 